\title{
Retraction Note to: Hop-Count Filtering: A Successful Security Alongside Spoofed Network Traffic
}

\author{
N.B. Pokale ${ }^{1}$ and Samuel S. Chopde ${ }^{2}$ \\ ${ }^{1}$ Asst. Professor - Department Information Technology \\ Sinhgad Academy of Engineering, Pune, India \\ nbpokale@gmail.com \\ ${ }^{2}$ Research Scholar - Department Information Technology \\ Sinhgad Academy of Engineering, Pune, India \\ samuel.chopdelgmail.com
}

DOI 10.1007/978-3-642-19542-6_132

The paper starting on page 535 of this volume has been retracted because a considerable amount of its content was plagiarized from the following publication:

"Hop-Count Filtering: An Effective Defense Against Spoofed DDoS Traffic" by Cheng Jin, Haining Wang, and Kang G. Shin, Copyright 2003 ACM 1-58113-7389/03/0010. 